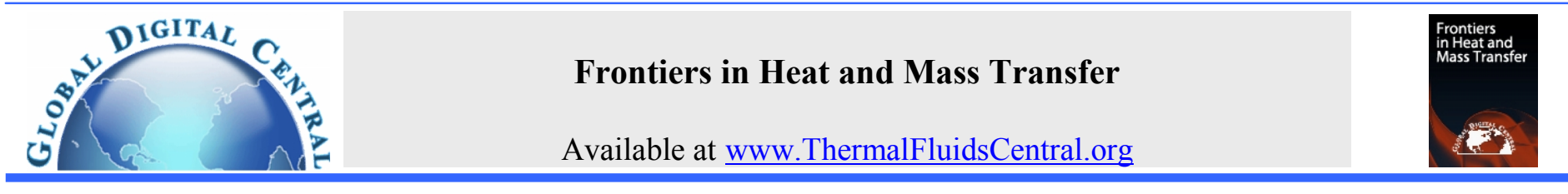

\title{
ON UNCERTAINTY AND LOCAL SENSITIVITY ANALYSIS FOR STEADY-STATE CONJUGATE HEAT TRANSFER PROBLEMS PART 1: EMISSIVITY, FLUID TEMPERATURE, AND CONDUCTANCE
}

\author{
Christian Rauch ${ }^{*}$ \\ VIRTUAL VEHICLE Research and Test Center (ViF), Graz, 8010, Austria
}

\begin{abstract}
In recent years, significant effort has been placed into developing automated multi-physics simulation. The exchange of boundary conditions has lead to more realistic as well as more complex simulations with usually slower convergence rate when the coupling is being performed between two different codes. In this paper the equations of local sensitivities for element centered steady-state combined convection, conduction, and thermal radiation problems are being derived. A numerical analysis on the stability of the solution matrix is being conducted. Partial uncertainties and the relative importance of the heat transfer modes are investigated by their uncertainty factors and conclusions are being drawn.
\end{abstract}

Keywords: Uncertainty Analysis, Conjugate Heat Transfer, Finite Difference Method, Steady-State, Thermal Radiation

\section{INTRODUCTION}

Conjugate heat transfer (CHT) problems have been the focus of research for many years. Often, various codes are being developed for different physical phenomena. There are several products on the market for Computational Fluid Dynamics (CFD) and thermal radiation, respectively. Since those codes are optimized for their respective models, segregated coupling becomes an interesting option. The exchange of boundary values between those codes, hopefully, will lead to a converged solution for all physical models involved. It is the skill of the simulation engineer to employ the most appropriate models, numerical schemes, and model parameters for those programs. Unfortunately, when performing those couplings, which can be quite time consuming, the engineer is left to decide, whether the results can be trusted. Usually, this is done by comparing simulation results with measurements. While finding an agreement with measurements is necessary, it does not guarantee the correctness of all the models in the whole parameter range. It might happen, that some model assumptions are erroneous, but at the specific measurement points those errors cancel out. This might cause accepted deviations in areas where measurements are not available, or for erroneously employing the models at different simulation settings where they could show unanticipated and possibly uncorrected deviations. In order to give the engineer more confidence in his results, a proper understanding of the influence of the model parameters on the system to be investigated is advisable. Sensitivity and uncertainty analyses are tools for providing more insight into the system.

Local sensitivity and uncertainty analysis for thermal radiation has not received as much attention as for conduction or convection. Blackwell et al. (1998) investigated a control volume finite element system with conjugate heat transfer, but they included only a simplified radiation equation for the interaction with the environment without the use of view factors and reflections within the geometry. To the best knowledge of the author, the first paper on thermal radiation analysis by uncertainty investigation with reflections between surfaces taken into account was published by Taylor et al. (1993). There, they investigated the sensitivities of view factors and emissivity when temperatures are given as boundary conditions. In later works (Taylor et al., 1994; Taylor et al., 1995), they expanded their research for changes in area and boundary conditions and for the case when a heat flux is specified instead of a temperature. One of their conclusions was that enforcing reciprocity and closure for view factors greatly improved accuracy. Taylor and Luck (1995) elaborated more on various methods of closure and reciprocity correction methods.

Korycki (2006) formulated sensitivity factors for CHT problems, including radiation in participating media, for the boundary element method (BEM).

Significant research on sensitivity analysis has been conducted in the area of structural mechanics. For example, Bhatia and Livne (2008) formulated sensitivity factors for the Finite Element Method (FEM). They employed those factors to estimate changing form factors due to mechanical and thermal deformations. In a follow-up paper (Bhatia and Livne, 2009) they presented a way of investigating temperature dependant parameters in a transient setting by reformulating the equations such, that those parameters were placed on the main diagonal.

This paper is a revised version published by Rauch and Almbauer (2010a) and is concerned with deriving sensitivity equations for the element-centered steady-state finite difference method. It can be regarded as a continuation of Taylors's work (Taylor et al., 1995) by expanding his approach for conduction and convection. In a related treatment, Rauch and Almbauer (2010) focused their work on sensitivities of view factors in CHT problems.

The next section derives the coefficients for emissivity, fluid temperature, and conductance followed by introducing the test case. Then, the results are analyzed for stability of the solution and the relative importance of heat transfer modes with and without partial uncertainties. Finally, conclusions are being drawn. 


\section{MATHEMATICAL FORMULATION}

At this point it is advantageous to introduce the thermal system. A thermal node $k$ consists of a center and a virtual extension. The node has an area $A_{k}$ where it can exchange energy with the surrounding. Optionally, the dashed line in Fig. 1 marks the border to the thermal back node. Through that border exchange from the surface node happens by conduction, only. The area $A_{k t}$ marks the area to the neighboring node $t$, where heat is transferred from the surface node $k$ to $t$ by conduction. The distance between two nodes is named $l_{k t}$.

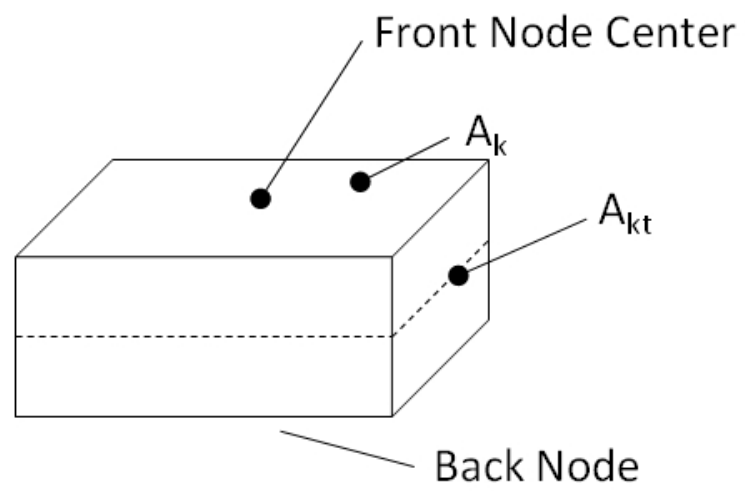

Fig. 1 Thermal Node

The formulation of local sensitivities is initiated by writing the radiative energy balance from Poljak's method (Siegel and Howell, 2002):

$q_{o, k}-\frac{1}{A_{k}} \sum_{s=1}^{N} A_{s} q_{o, s} F_{s k}=b_{k}$

Equation (1) states, that the thermal node $k$ emits energy and receives energy from all other nodes $N$ of the system. Here, $q_{o}$ is the radiosity, $F_{s k}$ the view factor from $s$ to $k$, and $b_{k}$ the boundary heat flux. For ease of derivation the first radiosity term is drawn into the summation:

$b_{k}=\sum_{s=1}^{N}\left[\delta_{s k} q_{o, s}-\delta_{s k} \frac{A_{s}}{A_{k}} q_{o, s} F_{s k}-\left(1-\delta_{s k}\right) \frac{A_{s}}{A_{k}} q_{o, s} F_{s k}\right]$

In principle, another formulation without radiosity could be employed but this would result in more difficulties in deriving the set of equations for sensitivities.

\subsection{Emissivity}

Now, the total derivatives of the parameters, here the emissivity $\varepsilon_{i}$, have to be calculated:

$$
\begin{aligned}
& \frac{d b_{k}}{d \varepsilon_{i}}=\sum_{s=1}^{N} \frac{\partial b_{k}}{\partial A_{s}} * \frac{\partial A_{s}}{\partial \varepsilon_{i}}+\sum_{s=1}^{N} \frac{\partial b_{k}}{\partial q_{o, s}} * \frac{\partial q_{o, s}}{\partial \varepsilon_{i}} \\
& +\sum_{s=1}^{N} \frac{\partial b_{k}}{\partial F_{s k}} * \frac{\partial F_{s k}}{\partial \varepsilon_{i}}+\sum_{s=1}^{N} \frac{\partial b_{k}}{\partial \varepsilon_{k}} * \frac{\partial \varepsilon_{k}}{\partial \varepsilon_{i}}
\end{aligned}
$$

The partial derivatives for the area and the view factor with respect to the emissivity can be ignored, as we do not consider changes in geometry due to thermal stresses. The partial derivative of the emissivity with respect to an emissivity is set to one when the two nodes are identical and zero, otherwise.

$$
\frac{\partial \varepsilon_{k}}{\partial \varepsilon_{i}}=1: k=i
$$

$\frac{\partial \varepsilon_{k}}{\partial \varepsilon_{i}}=0: k \neq i$

Though emissivity does not occur in Eq. (2), Eq. (16) will rely on Eq. (3) and thus will need the aforementioned partial derivatives with respect to emissivity. The radiosity will usually change with changing parameter:

$$
\frac{\partial b_{k}}{\partial q_{o, s}}=\sum_{s=1}^{N}\left[\delta_{k s}\left(1-F_{k k}\right)-\left(1-\delta_{k s}\right) \frac{A_{s}}{A_{k}} F_{s k}\right]
$$

Here, in the partial derivative of Eq. (3) with respect to the radiosity, the simplification has been used, that when the indices $s$ and $k$ are equal, the areas cancel. Substituting Eq. (6) into Eq. (3) with all the aforementioned simplifications yields:

$\frac{d b_{k}}{d \varepsilon_{i}}=\sum_{s=1}^{N}\left[\delta_{k s}\left(1-F_{k k}\right)-\left(1-\delta_{k s}\right) \frac{A_{s}}{A_{k}} F_{s k}\right] * \frac{\partial q_{o, s}}{\partial \varepsilon_{i}}$

Now, attention is turned to the boundary term $b_{k}$. In Taylor's work (Taylor et al., 1995) a specified temperature or heat flux had been used. When performing CHT calculations, the values of the heat transfer modes change and, consequently, also the node temperature $T_{k}$.

$b_{k}=h_{k}\left(T_{f, k}-T_{k}\right)+\frac{1}{A_{k}} \sum_{t=1}^{N_{\text {Cond }}}\left(-k_{k t} A_{k t} \frac{\left(T_{k}-T_{t}\right)}{l_{k t}}\right)$

Here, $h_{k}$ stands for the convective heat transfer coefficient, $T_{f, k}$ the fluid temperature next to the solid node $k$, and $k_{k t}$ the thermal conductivity from node $k$ to $t$. Also other heat loads could be included such as electrical heating or solar load. But in this work the emphasis is placed on the convection and conduction term. The summation runs over all neighboring nodes $N_{\text {Cond }}$.

$\frac{d b_{k}}{d \varepsilon_{i}}=\sum_{t=1}^{N_{\text {Cond }}} \frac{\partial b_{k}}{\partial T_{k}} * \frac{\partial T_{k}}{\partial \varepsilon_{i}}+\sum_{t=1}^{N_{\text {Cond }}} \frac{\partial b_{k}}{\partial T_{t}} * \frac{\partial T_{t}}{\partial \varepsilon_{i}}$

Again, the discussion is simplified by presuming the conductance $C_{k t}$ to be independent from temperature and emissivity:

$C_{k t}=\frac{k_{k t}}{l_{k t}} A_{k t}$

The convective heat transfer coefficient and the corresponding fluid temperature are assumed to be constant inputs from a CFD code and are thus treated as black box entries. Now, the two partial derivatives for $T_{k}$ and $\mathrm{T}_{\mathrm{t}}$ are shown:

$$
\begin{aligned}
& \frac{\partial b_{k}}{\partial T_{k}}=-h_{k}-\frac{1}{A_{k}} \sum_{t=1}^{N_{\text {Cond }}} \frac{k_{k t}}{l_{k t}} A_{k t} \\
& \frac{\partial b_{k}}{\partial T_{t}}=\frac{1}{A_{k}} \sum_{t=1}^{N_{\text {Cond }}}\left[\frac{k_{k t}}{l_{k t}} A_{k t}\right]
\end{aligned}
$$

Placing those last two equations into Eq. (9) one gets:

$\frac{d b_{k}}{d \varepsilon_{i}}=\left(-h_{k}-\frac{1}{A_{k}} \sum_{t=1}^{N_{\text {Cond }}} \frac{k_{k t}}{l_{k t}} A_{k t}\right) * \frac{\partial T_{k}}{\partial \varepsilon_{i}}+\frac{1}{A_{k}} \sum_{t=1}^{N_{\text {Cond }}}\left[\frac{k_{k t}}{l_{k t}} A_{k t}\right] * \frac{\partial T_{t}}{\partial \varepsilon_{i}}$ 
Equation (13) can now be substituted into Eq. (7):

$$
\begin{aligned}
& \sum_{s=1}^{N}\left[\delta_{k s}\left(1-F_{k k}\right)-\left(1-\delta_{k s}\right) \frac{A_{s}}{A_{k}} F_{s k}\right] * \frac{\partial q_{o, s}}{\partial \varepsilon_{i}} \\
& =\left(-h_{k}-\frac{1}{A_{k}} \sum_{t=1}^{N_{\text {Cond }}} \frac{k_{k t}}{l_{k t}} A_{k t}\right) * \frac{\partial T_{k}}{\partial \varepsilon_{i}}+\frac{1}{A_{k}} \sum_{t=1}^{N_{\text {Cond }}}\left[\frac{k_{k t}}{l_{k t}} A_{k t}\right] * \frac{\partial T_{t}}{\partial \varepsilon_{i}}
\end{aligned}
$$

Taylor et al. (1995) in their work were able to calculate the radiosity derivative, because there were no terms containing temperature derivatives. In Eq. (14) there are the partial derivative of temperature and radiosity as unknowns. Therefore, one needs a second equation, relating temperature to radiosity. Poljak's method provides one:

$q_{o, k}-\left(1-\varepsilon_{k}\right) \frac{1}{A_{k}} \sum_{s=1}^{N} A_{s} q_{o, s} F_{s k}=b_{k}$

This equation looks very similar to Eq. (1) except for the emissivity term. Again, the first radiosity term is put into the summation:

$$
\begin{aligned}
& b_{k}=\sum_{s=1}^{N}\left[\delta_{s k} q_{o, s}-\delta_{s k}\left(1-\varepsilon_{k}\right) \frac{A_{s}}{A_{k}} q_{o, s} F_{s k}\right. \\
& \left.-\left(1-\delta_{s k}\right)\left(1-\varepsilon_{k}\right) \frac{A_{s}}{A_{k}} q_{o, s} F_{s k}\right]
\end{aligned}
$$

The same reasoning is applied as for Eq. (3) along with the assumptions of Eqs. (4) and (5):

$$
\begin{aligned}
& \frac{\partial b_{k}}{\partial q_{o, s}}=\sum_{s=1}^{N}\left[\delta_{s k}-\delta_{s k}\left(1-\varepsilon_{k}\right) \frac{A_{s}}{A_{k}} F_{s k}-\left(1-\delta_{s k}\right)\left(1-\varepsilon_{k}\right) \frac{A_{s}}{A_{k}} F_{s k}\right] \\
& \frac{\partial b_{k}}{\partial \varepsilon_{k}}=\frac{1}{A_{k}} \sum_{s=1}^{N}\left[\delta_{s k} A_{s} q_{o, s} F_{s k}+\left(1-\delta_{s k}\right) A_{s} q_{o, s} F_{s k}\right]
\end{aligned}
$$

The last derivation can be simplified to:

$\frac{\partial b_{k}}{\partial \varepsilon_{k}}=\frac{1}{A_{k}} \sum_{s=1}^{N} A_{s} q_{o, s} F_{s k}$

Equations (17) and (19) are substituted into Eq. (3):

$$
\begin{aligned}
& \frac{d b_{k}}{d \varepsilon_{i}}=\sum_{s=1}^{N}\left[\delta_{k s}\left(1-\left(1-\varepsilon_{k}\right) F_{k k}\right)-\left(1-\delta_{k s}\right)\left(1-\varepsilon_{k}\right) \frac{A_{s}}{A_{k}} F_{s k}\right] \\
& * \frac{\partial q_{o, s}}{\partial \varepsilon_{i}}+\frac{\delta_{k i}}{A_{k}} \sum_{s=1}^{N} A_{s} q_{o, s} F_{s k}
\end{aligned}
$$

The right hand side of Eq. (15) states:

$$
b_{k}=\varepsilon_{k} \sigma T_{k}^{4}
$$

This term relates temperature to radiosity. Again, some partial derivatives are needed for Eq. (21):

$$
\begin{aligned}
& \frac{d b_{k}}{d \varepsilon_{i}}=\frac{\partial b_{k}}{\partial T_{k}} * \frac{\partial T_{k}}{\partial \varepsilon_{i}}+\frac{\partial b_{k}}{\partial \varepsilon_{k}} * \frac{\partial \varepsilon_{k}}{\partial \varepsilon_{i}} \\
& \frac{\partial b_{k}}{\partial \varepsilon_{k}}=\sigma T_{k}^{4}
\end{aligned}
$$

$\frac{\partial b_{k}}{\partial T_{k}}=4 \varepsilon_{k} \sigma T_{k}^{3}$

When substituting Eqs. (23) and (24) into Eq. (22) along with the assumptions of Eqs. (4) and (5) one obtains the following relation:

$\frac{d b_{k}}{d \varepsilon_{i}}=4 \varepsilon_{k} \sigma T_{k}^{3} \frac{\partial T_{k}}{\partial \varepsilon_{i}}+\sigma T_{k}^{4} \frac{\partial \varepsilon_{k}}{\partial \varepsilon_{i}}=4 \varepsilon_{k} \sigma T_{k}^{3} \frac{\partial T_{k}}{\partial \varepsilon_{i}}+\delta_{i k} \sigma T_{k}^{4}$

Equaling Eq. (25) with Eq. (20) one gets:

$\sum_{s=1}^{N}\left[\delta_{k s}\left(1-\left(1-\varepsilon_{k}\right) F_{k k}\right)-\left(1-\delta_{k s}\right)\left(1-\varepsilon_{k}\right) \frac{A_{s}}{A_{k}} F_{s k}\right]$

$* \frac{\partial q_{o, s}}{\partial \varepsilon_{i}}+\frac{\delta_{k i}}{A_{k}} \sum_{s=1}^{N} A_{s} q_{o, s} F_{s k}=4 \varepsilon_{k} \sigma T_{k}^{3} \frac{\partial T_{k}}{\partial \varepsilon_{i}}+\delta_{i k} \sigma T_{k}^{4}$

This is a second equation for the unknown derivatives in temperature and radiosity with respect to emissivity. Eq. (26) is rewritten to explicitly calculate the temperature derivative:

$$
\begin{aligned}
& \frac{\partial T_{k}}{\partial \varepsilon_{i}}=\left[\sum_{s=1}^{N}\left[\delta_{k s}\left(1-\left(1-\varepsilon_{k}\right) F_{k k}\right)-\left(1-\delta_{k s}\right)\left(1-\varepsilon_{k}\right) \frac{A_{s}}{A_{k}} F_{s k}\right]\right. \\
& \left.* \frac{\partial q_{o, s}}{\partial \varepsilon_{i}}+\frac{\delta_{k i}}{A_{k}} \sum_{s=1}^{N} A_{s} q_{o, s} F_{s k}-\delta_{i k} \sigma T_{k}^{4}\right] \frac{1}{4 \varepsilon_{k} \sigma T_{k}^{3}}
\end{aligned}
$$

When the index $k$ is replaced with index $t$ in Eq. (27) one gets:

$$
\begin{aligned}
& \frac{\partial T_{t}}{\partial \varepsilon_{i}}=\left[\sum_{s=1}^{N}\left[\delta_{t s}\left(1-\left(1-\varepsilon_{t}\right) F_{t t}\right)-\left(1-\delta_{t s}\right)\left(1-\varepsilon_{t}\right) \frac{A_{s}}{A_{t}} F_{s t}\right]\right. \\
& \left.* \frac{\partial q_{o, s}}{\partial \varepsilon_{i}}+\frac{\delta_{t i}}{A_{t}} \sum_{s=1}^{N} A_{s} q_{o, s} F_{s t}-\delta_{i t} \sigma T_{t}^{4}\right] \frac{1}{4 \varepsilon_{t} \sigma T_{t}^{3}}
\end{aligned}
$$

One can substitute Eqs. (27) and (28) into Eq. (14):

$$
\begin{aligned}
& \sum_{s=1}^{N}\left[\delta_{k s}\left(1-F_{s k}\right)-\left(1-\delta_{k s}\right) \frac{A_{s}}{A_{k}} F_{s k}\right] * \frac{\partial q_{o, s}}{\partial \varepsilon_{i}}=\left(-h_{k}-\frac{1}{A_{k}} \sum_{t=1}^{N_{\text {Cond }}} \frac{k_{k t}}{l_{k t}} A_{k t}\right) \\
& * \frac{1}{4 \varepsilon_{k} \sigma T_{k}^{3}}\left(\sum_{s=1}^{N}\left[\delta_{k s}\left(1-\left(1-\varepsilon_{k}\right) F_{s k}\right)-\left(1-\delta_{k s}\right)\left(1-\varepsilon_{k}\right) \frac{A_{s}}{A_{k}} F_{s k}\right] * \frac{\partial q_{o, s}}{\partial \varepsilon_{i}}\right. \\
& \left.+\frac{\delta_{k i}}{A_{k}} \sum_{s=1}^{N} A_{s} q_{o, s} F_{s k}-\delta_{i k} \sigma T_{k}^{4}\right)+\frac{1}{A_{k}} \sum_{t=1}^{N_{\text {Cond }}}\left[\frac{k_{k t}}{l_{k t}} A_{k t}\right] \\
& * \frac{1}{4 \varepsilon_{t} \sigma T_{t}^{3}}\left(\sum_{s=1}^{N}\left[\delta_{t s}\left(1-\left(1-\varepsilon_{t}\right) F_{s t}\right)-\left(1-\delta_{t s}\right)\left(1-\varepsilon_{t}\right) \frac{A_{s}}{A_{t}} F_{s t}\right] * \frac{\partial q_{o, s}}{\partial \varepsilon_{i}}\right. \\
& \left.+\frac{\delta_{t i}}{A_{t}} \sum_{s=1}^{N} A_{s} q_{o, s} F_{s t}-\delta_{i t} \sigma T_{t}^{4}\right)
\end{aligned}
$$

After putting all the terms with the radiosity derivative on the left hand side the following final relation has been derived as it was actually implemented:

$$
\begin{aligned}
& \sum_{s=1}^{N}\left[\delta_{k s}\left(1-F_{k k}\right)-\left(1-\delta_{k s}\right) \frac{A_{s}}{A_{k}} F_{s k}\right] * \frac{\partial q_{o, s}}{\partial \varepsilon_{i}} \\
& -\left(-h_{k}-\frac{1}{A_{k}} \sum_{t=1}^{N_{\text {Cond }}} \frac{k_{k t}}{l_{k t}} A_{k t}\right) * \frac{1}{4 \varepsilon_{k} \sigma T_{k}^{3}} \\
& * \sum_{s=1}^{N}\left[\delta_{k s}\left(1-\left(1-\varepsilon_{k}\right) F_{k k}\right)-\left(1-\delta_{k s}\right)\left(1-\varepsilon_{k}\right) \frac{A_{s}}{A_{k}} F_{s k}\right]
\end{aligned}
$$




$$
\begin{aligned}
& * \frac{\partial q_{o, s}}{\partial \varepsilon_{i}}-\frac{1}{A_{k}} \sum_{t=1}^{N_{\text {Cond }}}\left[\frac{k_{k t}}{l_{k t}} A_{k t}\right] * \frac{1}{4 \varepsilon_{t} \sigma T_{t}^{3}} \\
& * \sum_{s=1}^{N}\left[\delta_{t s}\left(1-\left(1-\varepsilon_{t}\right) F_{t t}\right)-\left(1-\delta_{t s}\right)\left(1-\varepsilon_{t}\right) \frac{A_{s}}{A_{t}} F_{s t}\right] * \frac{\partial q_{o, s}}{\partial \varepsilon_{i}} \\
& =\left(-h_{k}-\frac{1}{A_{k}} \sum_{t=1}^{N_{\text {Cond }}} \frac{k_{k t}}{l_{k t}} A_{k t}\right) * \frac{1}{4 \varepsilon_{k} \sigma T_{k}^{3}} \\
& *\left(\frac{\delta_{k i}}{A_{k}} \sum_{s=1}^{N} A_{s} q_{o, s} F_{s k}-\delta_{i k} \sigma T_{k}^{4}\right) \\
& +\frac{1}{A_{k}} \sum_{t=1}^{N_{\text {Cond }}}\left[\frac{k_{k t}}{l_{k t}} A_{k t}\right] * \frac{1}{4 \varepsilon_{t} \sigma T_{t}^{3}} *\left(\frac{\delta_{t i}}{A_{t}} \sum_{s=1}^{N} A_{s} q_{o, s} F_{s t}-\delta_{i t} \sigma T_{t}^{4}\right)
\end{aligned}
$$

Once the radiosity derivative has been calculated by Eq. (30), one can simply multiply the results vector by Eq. (27).

If the node temperature is given as a boundary condition, Eq. (27) can be taken instead of Eq. (30). Since the derivative of node temperature with respect to emissivity will be zero in that particular case the change of radiosity with respect to emissivity can be calculated by the following equation:

$$
\begin{aligned}
& \sum_{s=1}^{N}\left[\delta_{k s}\left(1-\left(1-\varepsilon_{k}\right) F_{s k}\right)-\left(1-\delta_{k s}\right)\left(1-\varepsilon_{k}\right) \frac{A_{s}}{A_{k}} F_{s k}\right] * \frac{\partial q_{o, s}}{\partial \varepsilon_{i}} \\
& =-\frac{\delta_{k i}}{A_{k}} \sum_{s=1}^{N} A_{s} q_{o, s} F_{s k}+\delta_{i k} \sigma T_{k}^{4}
\end{aligned}
$$

With the radiosity derivative with respect to emissivity known, Eq. (7) can be used to calculate the change in net heat flux on that node.

\subsection{Fluid Temperature}

The mathematical derivation for the change in radiosity with respect to the fluid temperature $T_{f}$ is very similar. Instead of Eqs. (3) and (7), one has:

$$
\begin{aligned}
& \frac{d b_{k}}{d T_{f, i}}=\sum_{s=1}^{N} \frac{\partial b_{k}}{\partial q_{o, s}} * \frac{\partial q_{o, s}}{\partial T_{f, i}} \\
& \frac{d b_{k}}{d T_{f, i}}=\sum_{s=1}^{N}\left[\delta_{k s}\left(1-F_{k k}\right)-\left(1-\delta_{k s}\right) \frac{A_{s}}{A_{k}} F_{s k}\right] * \frac{\partial q_{o, s}}{\partial T_{f, i}}
\end{aligned}
$$

For the partial derivatives of Eq. (8) we now have to add a term for the derivation in $\mathrm{T}_{\mathrm{f}}$

$$
\begin{aligned}
\frac{d b_{k}}{d T_{f, i}} & =\sum_{t=1}^{N_{\text {Cond }}} \frac{\partial b_{k}}{\partial T_{k}} * \frac{\partial T_{k}}{\partial T_{f, i}}+\sum_{t=1}^{N_{\text {Cond }}} \frac{\partial b_{k}}{\partial T_{t}} * \frac{\partial T_{t}}{\partial T_{f, i}}+\frac{\partial b_{k}}{\partial T_{f, k}} * \frac{\partial T_{f, k}}{\partial T_{f, i}} \\
\frac{\partial T_{f, k}}{\partial T_{f, i}} & =1: i=k \\
\frac{\partial T_{f, k}}{\partial T_{f, i}} & =0: i \neq k
\end{aligned}
$$

The expressions in Eqs. (35) and (36) were derived under the assumption, that heat transfer coefficients are treated as black box entries. Therefore, only the value of $T_{f}$ at node $i$ has a derivation in the form of the convective heat transfer coefficient. At all other nodes the derivation is zero. When abandoning the black box assumption, one could argue, that changing a fluid temperature at one node could change the fluid temperatures at all other nodes. This could happen in a closed cavity flow. In that case, the index on the right hand side of Eq. (35) would hold the index $k$ as was published in Rauch and Almbauer (2010a). In this paper the assumption of general flow is followed and Eq. (35) holds.
Along with Eqs. (11) and (12) one gets:

$$
\begin{aligned}
& \frac{d b_{k}}{d T_{f, i}}=\left(-h_{k}-\frac{1}{A_{k}} \sum_{t=1}^{N_{\text {Cond }}} \frac{k_{k t}}{l_{k t}} A_{k t}\right) * \frac{\partial T_{k}}{\partial T_{f, i}} \\
& +\frac{1}{A_{k}} \sum_{t=1}^{N_{\text {Cond }}}\left[\frac{k_{k t}}{l_{k t}} A_{k t}\right] * \frac{\partial T_{t}}{\partial T_{f, i}}+\delta_{i k} h_{i}
\end{aligned}
$$

Because the procedure for obtaining the radiosity and temperature gradients follow the same procedure as for emissivity, only those equations are reproduced as deemed necessary for use and understanding of this text.

In the same way as for Eq. (27), the temperature gradient with respect to fluid temperature is expressed as:

$$
\begin{aligned}
& \frac{\partial T_{k}}{\partial T_{f, i}}=\frac{1}{4 \varepsilon_{k} \sigma T_{k}^{3}} \\
& *\left(\sum_{s=1}^{N}\left[\delta_{k s}\left(1-\left(1-\varepsilon_{k}\right) F_{s k}\right)-\left(1-\delta_{k s}\right)\left(1-\varepsilon_{k}\right) \frac{A_{s}}{A_{k}} F_{s k}\right] * \frac{\partial q_{o, s}}{\partial T_{f, i}}\right)
\end{aligned}
$$

The final relation is derived for the first order fluid temperature gradient:

$$
\begin{aligned}
& \sum_{s=1}^{N}\left[\delta_{k s}\left(1-F_{k k}\right)-\left(1-\delta_{k s}\right) \frac{A_{s}}{A_{k}} F_{s k}\right] * \frac{\partial q_{o, s}}{\partial T_{f, i}} \\
& -\left(-h_{k}-\frac{1}{A_{k}} \sum_{t=1}^{N_{C o n d}} \frac{k_{k t}}{l_{k t}} A_{k t}\right) * \frac{1}{4 \varepsilon_{k} \sigma T_{k}^{3}} \\
& * \sum_{s=1}^{N}\left[\delta_{k s}\left(1-\left(1-\varepsilon_{k}\right) F_{k k}\right)-\left(1-\delta_{k s}\right)\left(1-\varepsilon_{k}\right) \frac{A_{s}}{A_{k}} F_{s k}\right] * \frac{\partial q_{o, s}}{\partial T_{f, i}} \\
& -\frac{1}{A_{k}} \sum_{t=1}^{N_{C o n d}}\left[\frac{k_{k t}}{l_{k t}} A_{k t}\right] * \frac{1}{4 \varepsilon_{t} \sigma T_{t}^{3}} \\
& * \sum_{s=1}^{N}\left[\delta_{t s}\left(1-\left(1-\varepsilon_{t}\right) F_{t t}\right)-\left(1-\delta_{t s}\right)\left(1-\varepsilon_{t}\right) \frac{A_{s}}{A_{t}} F_{s t}\right] * \frac{\partial q_{o, s}}{\partial T_{f, i}}=\delta_{i k} h_{i}
\end{aligned}
$$

Once the radiosity derivative has been calculated by Eq. (39), one can simply multiply the result vector by Eq. (38).

If the node temperature and all neighboring node temperatures are given as a boundary condition, Eq. (37) can be taken instead of Eq. (39). Since the derivative of node temperature with respect to emissivity will be zero in that particular case the change of radiosity with respect to the fluid temperature can be calculated by the following equation:

$$
\sum_{s=1}^{N}\left[\delta_{k s}\left(1-F_{s k}\right)-\left(1-\delta_{k s}\right) \frac{A_{s}}{A_{k}} F_{s k}\right] * \frac{\partial q_{o, s}}{\partial T_{f, i}}=\delta_{i k} h_{i}
$$

With the radiosity derivative with respect to fluid temperature known, Eq. (33) can be used to calculate the change in net heat flux on that node.

\subsection{Conductance}

Finally, the equation for the conductance derivative shall be shown. The conductance was defined in Eq. (10). The reason for using this lumped property is the ease with which it can be retrieved from the thermal radiation solver employed in this study. Starting from Eq. (2) one derives with Eq. (6):

$$
\frac{d b_{k}}{d C_{i j}}=\sum_{s=1}^{N}\left[\delta_{k s}\left(1-F_{k k}\right)-\left(1-\delta_{k s}\right) \frac{A_{s}}{A_{k}} F_{s k}\right] * \frac{\partial q_{o, s}}{\partial C_{i j}}
$$


Eq. (10) is substituted into Eq. (8):

$$
b_{k}=q_{i m p}+h_{k}\left(T_{f, k}-T_{k}\right)+\frac{1}{A_{k}} \sum_{t=1}^{N_{\text {Cond }}}\left(-C_{k t} *\left(T_{k}-T_{t}\right)\right)
$$

When changing a conductance from $i$ to $j$ the conductance from $j$ to $i$ will also change. Otherwise, the derivation will be zero:

$$
\frac{\partial C_{k t}}{\partial C_{i j}}=1:(i=k \wedge j=t) \vee(j=k \wedge i=t)
$$

Using the above reasoning and the same procedure as for emissivity one arrives at the following equations:

$$
\begin{aligned}
& \frac{d b_{k}}{d C_{i j}}=\left(-h_{k}-\frac{1}{A_{k}} \sum_{t=1}^{N_{\text {Cond }}} C_{k t}\right) * \frac{\partial T_{k}}{\partial C_{i j}}+\frac{1}{A_{k}} \sum_{t=1}^{N_{\text {cond }}}\left(C_{k t} * \frac{\partial T_{t}}{\partial C_{i j}}\right) \\
& -\frac{1}{A_{k}} \sum_{t=1}^{N_{\text {Cond }}}\left[\left(T_{k}-T_{t}\right)\left(\delta_{i k} \delta_{j t}+\delta_{i t} \delta_{j k}\right)\right] \\
& \sum_{s=1}^{N}\left[\delta_{s k}-\delta_{s k}\left(1-\varepsilon_{k}\right) F_{s k}-\left(1-\delta_{s k}\right)\left(1-\varepsilon_{k}\right) \frac{A_{s}}{A_{k}} F_{s k}\right] * \frac{\partial q_{o, s}}{\partial C_{i j}} \\
& =4 \varepsilon_{k} \sigma T_{k}^{3} \frac{\partial T_{k}}{\partial C_{i j}}
\end{aligned}
$$

Eq. (45) is reformulated to express the temperature gradient and finally yields the equation for the radiosity gradient with respect to conductance:

$$
\begin{aligned}
& \sum_{s=1}^{N}\left[\delta_{k s}\left(1-F_{s k}\right)-\left(1-\delta_{k s}\right) \frac{A_{s}}{A_{k}} F_{s k}\right] * \frac{\partial q_{o, s}}{\partial C_{i j}} \\
& \left(-h_{k}-\frac{1}{A_{k}} \sum_{t=1}^{N_{\text {Cond }}} C_{k t}\right) * \frac{1}{4 \varepsilon_{k} \sigma T_{k}^{3}} \\
& * \sum_{s=1}^{N}\left[\delta_{s k}-\delta_{s k}\left(1-\varepsilon_{k}\right) F_{s k}-\left(1-\delta_{s k}\right)\left(1-\varepsilon_{k}\right) \frac{A_{s}}{A_{k}} F_{s k}\right] * \frac{\partial q_{o, s}}{\partial C_{i j}} \\
& -\frac{1}{A_{k}} \sum_{t=1}^{N_{\text {Cond }}}\left(C _ { k t } * \frac { 1 } { 4 \varepsilon _ { t } \sigma T _ { t } ^ { 3 } } \sum _ { s = 1 } ^ { N } \left[\delta_{s t}-\delta_{s t}\left(1-\varepsilon_{t}\right) F_{s t}\right.\right. \\
& \left.\left.-\left(1-\delta_{s t}\right)\left(1-\varepsilon_{t}\right) \frac{A_{s}}{A_{t}} F_{s t}\right] * \frac{\partial q_{o, s}}{\partial C_{i j}}\right) \\
& =-\frac{1}{A_{k}} \sum_{t=1}^{N_{\text {Cond }}}\left[\left(T_{k}-T_{t}\right)\left(\delta_{i k} \delta_{j t}+\delta_{i t} \delta_{j k}\right)\right]
\end{aligned}
$$

The reader should note that on the right-hand side (RHS) there will be two entries as long as there is one neighboring node. Once the radiosity derivative has been calculated by Eq. (46), one can simply multiply the results vector by Eq. (45).

If the node temperature and all neighboring node temperatures are given as a boundary condition, Eq. (44) can be used straight for calculating the change of heat flux. For mixed boundary condition settings Eq. (46) has to be used along with Eq. (41) for the fixed temperature at node $k$.

\subsection{Uncertainty}

The combined standard uncertainty $U_{c}$ can be formulated as follows:

$U_{c}=\sqrt{\sum_{s=1}^{N}\left(\frac{\varepsilon_{s}}{T_{k}} \frac{\partial T_{k}}{\partial \varepsilon_{s}}\right)^{2}} u_{\varepsilon}+\sqrt{\sum_{s=1}^{N}\left(\frac{T_{f, s}}{T_{k}} \frac{\partial T_{k}}{\partial T_{f, s}}\right)^{2}} u_{T_{f}}$
$+\sqrt{\sum_{s=1}^{N} \sum_{t=1}^{N_{C o n d}}\left(\frac{C_{s t}}{T_{k}} \frac{\partial T_{k}}{\partial C_{s t}}\right)^{2}} u_{C}$

The values with square roots are uncertainty factors, which are calculated by adding the sensitivity factors of the previous sections. It is common practice to publish them in a dimensionless way. For comparison purposes these factors are here normalized. This contrasts to the work by Taylor et al. (1995) where view factors and emissivities were not normalized because they are supposed to be no greater than one. In the present author's experience for view factors this is not necessarily guaranteed. The standard uncertainty $u$ uses either measurements or reflects expert knowledge. As this is usually not available for every node, this property is placed outside the square root. Alternatively, one could determine those uncertainties for regions and split the uncertainty factors accordingly.

\subsection{Work Flow}

The method proposed here is a posteriori because first, a conjugate heat transfer case needs to be calculated. The results of this simulation such as node temperature and radiosities need to be extracted along with geometric information, boundary conditions, and view factors in order to calculate the radiosity gradients as described in Eqs. (30), (39), and (46). Because this has to be done for a change in every node, LU factorization has been employed. In this way, all radiosity gradients are obtained by vector matrix multiplication. Once those radiosity gradients are known, temperature gradients described in Eqs. (27), (38), and (45) can be calculated. These gradients are used in Eq. (47) after rendering them dimensionless. Along with standard uncertainties actual temperature ranges can be provided by multiplying the combined standard uncertainty by the node temperature.

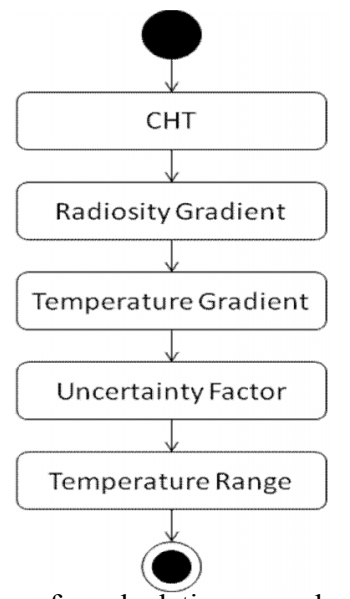

Fig. 2 Activity diagram for calculation procedure.

\section{Test Case}

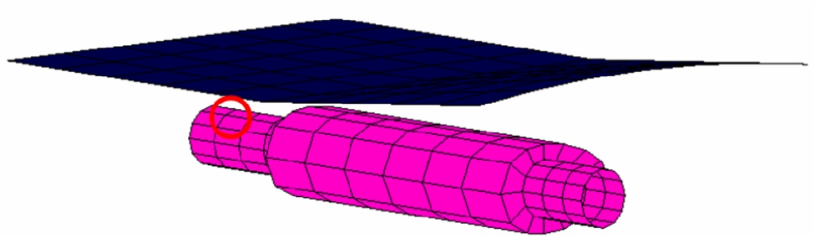

Fig. 3 Muffler geometry.

A generic muffler without internals serves as an example. The geometry consists of 260 quadrilaterals. The muffler is topped by a heat shield which is insulated at the back.

The case is set up in the commercial radiation solver RadThermIR 9.1. A built-in heat transfer model was used to provide convective heat transfer coefficients. The exterior boundary conditions were chosen in a way that would be similar to a highway drive. They are shown in the following table. 
The form factors were calculated with 512 rays per face. The exhaust gas is modeled as air without soot formation and non-participating for radiation. Though severe simplifications, the exhaust gas serves merely as a heat source. The exhaust gas itself is held at a constant temperature. Further details can be found in table 1.

Table 1 Boundary conditions for the muffler.

\begin{tabular}{|c|c|c|c|c|}
\hline \multirow{2}{*}{ Entity } & \multirow{2}{*}{ Unit } & \multirow{2}{*}{ Heat Shield } & \multicolumn{2}{|c|}{ Muffler } \\
\cline { 4 - 5 } & & & Outside & Inside \\
\hline Emissivity & {$[-]$} & 0,6 & 0,22 & 0,9 \\
\hline Thickness & {$[\mathrm{m}]$} & 0,002 & 0,001 & 0,001 \\
\hline Conductivity & {$[\mathrm{W} / \mathrm{m} / \mathrm{K}]$} & 17,3056 & 52,019 & 52,019 \\
\hline Air Velocity & {$[\mathrm{m} / \mathrm{s}]$} & 20 & 20 & 20 \\
\hline Air Temperature & {$[\mathrm{K}]$} & 303 & 303 & 413 \\
\hline
\end{tabular}

Table 2 provides the resulting net heat fluxes for the thermal node indicated in Fig. 3 by the red circle.

Table 2 Net heat fluxes for node 96 .

\begin{tabular}{|c|c|}
\hline Node & 96 \\
\hline & {$\left[\mathrm{W} / \mathrm{m}^{2}\right]$} \\
\hline Radiation & $-91,08$ \\
\hline Convection & $-3.598,71$ \\
\hline Conduction & $3.688,99$ \\
\hline
\end{tabular}

The results indicate that conduction plays the most important role for that node and radiation is insignificant.

\section{Numerical Analysis}

A numerical analysis has been conducted, because the author experienced severe difficulties in convergence behavior when employing an iterative solver. The Gerschgorin circle theorem helps in estimating eigenvalues $\lambda$ and thus stability of a solver because it gives the maximum possible value of $\lambda$ by the Gerschgorin radius $r$. The eigenvalue $\lambda$ assures stability if it is less than or equal to 1 . This is guaranteed when the radius $r$ is less than or equal 0.5 . In that case it can be expected, that the set of equations can be handled by an iterative solver without preconditioning.

$r=\sum_{\substack{j=1 \\ j \neq i}}^{N}\left|a_{i j}\right|$

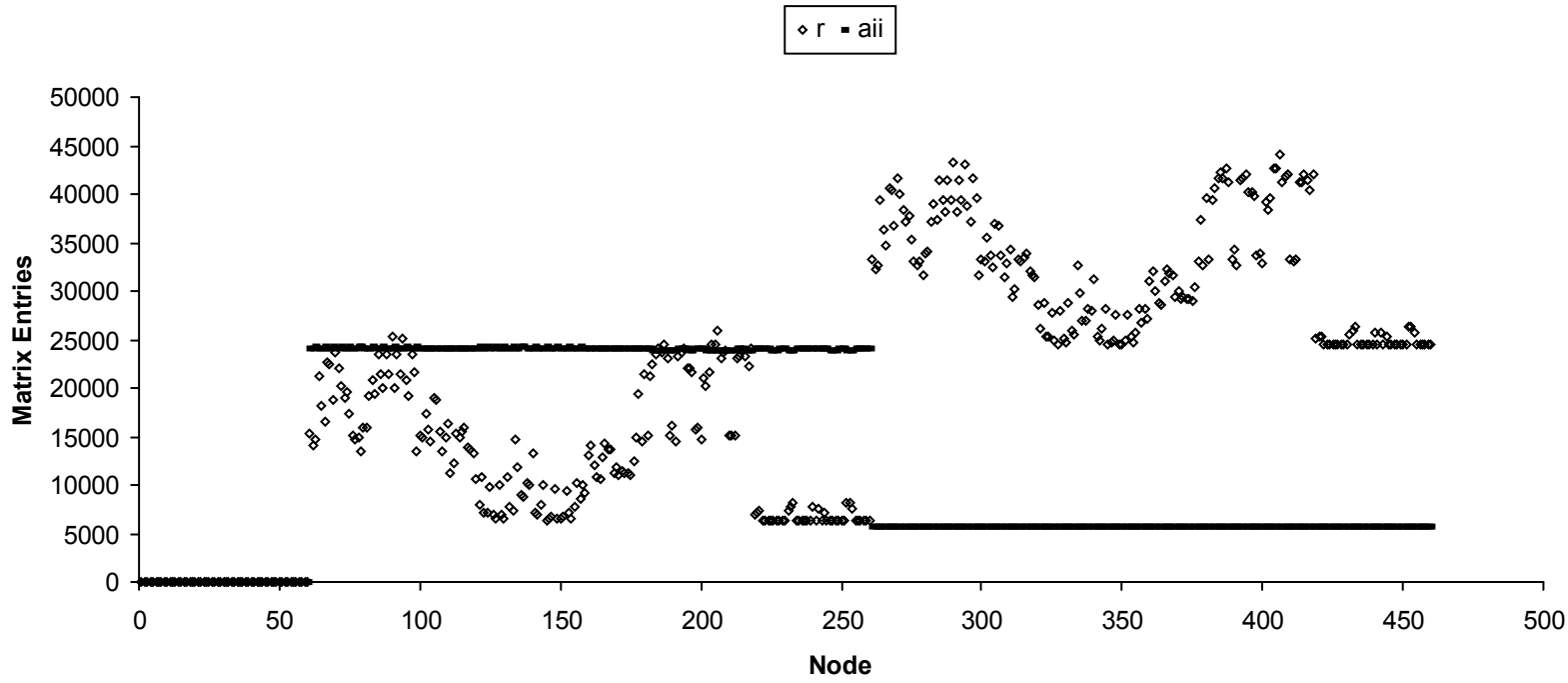

Fig. 4 Matrix entries and Gerschgorin radii of the muffler with all three heat transfer modes.
Here, $a_{i j}$ is a matrix entry of row $i$ and column $j$. It should be noted that in Eqs. (30), (39), and (46) the RHS are all identical. They only differ on their left hand side (LHS).

Figure 4 shows the diagonal $a_{i i}$ and Gerschgorin radii $r$ entries of the solution matrix $\boldsymbol{M}$, when all three heat transfer modes are included. In Rauch and Almbauer (2010a) figures are shown with radiation only, radiation and convection, and radiation and conduction terms included.

The figure presented here differs only marginally from the one with radiation and conduction terms only. The first 60 nodes constitute the heat shield. The next 200 nodes belong to the exterior of the muffler, and the last 200 nodes are ascribed to the inside of the muffler.

Figure 4 shows, that the radii are not only exceeding 1, but also that the node entries at the interior of the muffler are not diagonally dominant. Thus, this system is ill-conditioned.

The infinity norm $\|M\|_{\infty}$ of the matrix $\boldsymbol{M}$ is another indicator for stability.

$\|\mathbf{M}\|_{\infty}=\max _{1<i<N} \sum_{j=1}^{N}\left|a_{i j}\right|$

The value of 49915.7 is again very large, hinting for instability. In order to achieve a solution, the suggestion of Taylor et al. (1995) was followed by implementing an LU factorization algorithm. For large industrial models a parallelized distributed memory version would be required. Otherwise, an iterative solver would be needed, and hence, there would be a need for a suitable preconditioner.

Partial uncertainty calculation usually is practiced when calculating certain differentials becomes too cumbersome. Though this is not applicable in this case, it serves as a demonstration how interactions between the parameters have been taken care of.

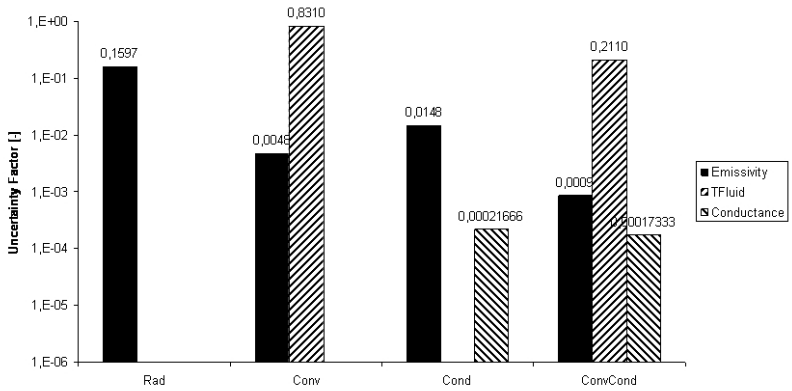

Fig. 5 Partial uncertainty calculation for node 96 of the muffler. 
The first column in Fig. 5 has been calculated with the radiation term, only. Emissivity exhibits a value well below one, indicating that its influence might not be dominant. The second column deals with a calculation including radiation and convection terms. Fluid temperature clearly shows its prominent role for that node. Emissivity dropped by two orders of magnitude. When considering the radiation and conduction terms as shown in the third column of Fig. 5, the uncertainty factor UF for emissivity dominates by two orders of magnitude. Considering all heat transfer modes yields the last column of Fig. 5.

Now, emissivity becomes insignificant by three orders of magnitude compared to the fluid temperature. This result was expected by the heat flux magnitude in Table 2. But the UF in fluid temperature also dominates the one in conductance. This result cannot be derived from heat flux analysis alone. The aim of sensitivity analysis is the ability of a model to change its state. It is noteworthy, that emissivity and, to some extent, conductance show a large sensitive behavior. Without taking into account all three heat transfer modes erroneous results are possible.

It should be mentioned, that the equations derived in this paper are based on radiosity. Therefore, thermal radiation cannot be excluded from consideration. In thermal radiation solvers it is usually possible to exclude nodes from radiation as a means of speeding up calculations. In that case, sensitivities in conductance and fluid temperature cannot be treated with the equations presented.

Finally, Fig. 6 shows uncertainty factors for conductance of the muffler.

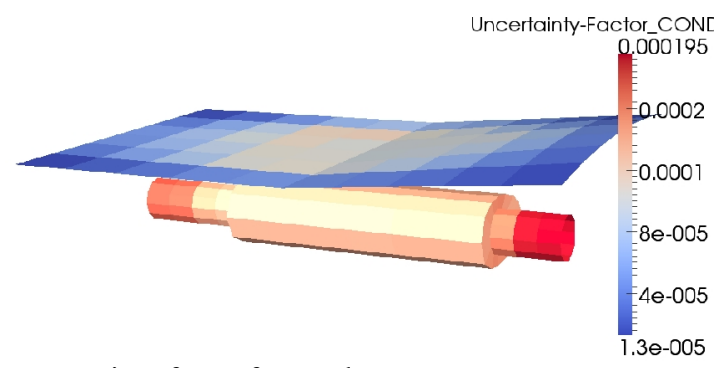

Fig. 6 Uncertainty factor for conductance.

Plotting these values on the surface gives insight on where a property change has a more profound effect, aiding the thermal engineer in the choice of the appropriate parameters for change in order to reduce deviations to measurements, or improving confidence in results.

\section{CONCLUSIONS}

The equations for sensitive factors were derived for emissivity, fluid temperature, and conductance, representing all three heat transfer modes. With these formulations the effects of those parameters on the total system can be explored. They can give better hints on what submodel needs to be refined in conjugate heat transfer problems. Partial uncertainties should be avoided in order to not miss the interactions of those parameters.

\section{ACKNOWLEDGEMENTS}

The author would like to acknowledge the financial support of the "COMET K2 - Competence Centres for Excellent Technologies Programme" of the Austrian Federal Ministry for Transport, Innovation and Technology (BMVIT), the Austrian Federal Ministry of Economy, Family and Youth (BMWFJ), the Austrian Research Promotion Agency (FFG), the Province of Styria and the Styrian Business Promotion Agency (SFG). I would furthermore like to express my thanks to my supporting scientific partner Graz University of Technology.

\section{NOMENCLATURE}

\begin{tabular}{ll} 
a & matrix entity \\
A & area $\left(\mathrm{m}^{2}\right)$ \\
C & conductance $(\mathrm{W} / \mathrm{K})$ \\
$\mathrm{F}$ & view factor $(-)$ \\
$\mathrm{h}$ & convective heat transfer coefficient $\left(\mathrm{W} / \mathrm{m}^{2} \cdot \mathrm{K}\right)$ \\
$\mathrm{k}$ & thermal conductivity $(\mathrm{W} / \mathrm{m} \cdot \mathrm{K})$ \\
$\mathrm{l}$ & distance between node centers $(\mathrm{m})$ \\
M & matrix \\
$\mathrm{N}$ & number of elements \\
$\mathrm{q}$ & heat flux $\left(\mathrm{W} / \mathrm{m}^{2}\right)$ \\
$\mathrm{r}$ & radius of Gerschgorin theorem \\
$\mathrm{T}$ & temperature $(\mathrm{K})$ \\
$\mathrm{u}$ & standard uncertainty $(-)$ \\
$\mathrm{U}$ & uncertainty $(-)$ \\
$\mathrm{UF}$ & uncertainty factor $(-)$ \\
$\|\cdot\|$ & norm \\
$|\cdot|$ & absolute value \\
Greek Symbols \\
$\delta$ & Kronecker delta $(-)$ \\
$\lambda$ & eigenvalue $(-)$ \\
$\varepsilon$ & total emissivity $(-)$ \\
$\sigma$ & Stefan-Boltzmann constant $\left(\mathrm{W} / \mathrm{m}^{2} \cdot \mathrm{K}^{4}\right)$ \\
Subscripts & \\
$\mathrm{b}$ & boundary heat flux \\
$\mathrm{c}$ & combined \\
C & conductance \\
Cond & conduction \\
$\mathrm{f}$ & fluid \\
$\mathrm{i}, \mathrm{j}$ & index from element i to $\mathrm{j}$ \\
$\mathrm{k}$ & index of element \\
$\mathrm{q}$ & heat flux specified \\
$\mathrm{s}, \mathrm{t}$ & running index \\
$\mathrm{T}$ & temperature specified \\
$\varepsilon$ & emissivity \\
$\infty$ & infinity \\
& \\
\hline &
\end{tabular}

\section{REFERENCES}

Bhatia, M., and Livne, E., 2008, "Design-Oriented Thermostructural Analysis with External and Internal Radiation, Part 1: Steady State," AIAA Journal, 46(3), 578-590.

http://dx.doi.org/10.2514/1.26236

Bhatia, M., and Livne, E., 2009, "Design-Oriented Thermostructural Analysis with External and Internal Radiation, Part 2: Transient Response," AIAA Journal, 47(5), 1228-1240.

http://dx.doi.org/10.2514/1.40265

Blackwell, B., Dowding, K., Cochran, R., and Dobranich, D., 1998, "Utilization of Sensitivity Coefficients to Guide the Design of a Thermal Battery," IMECE-981107, ASME 1998 International Mechanical Engineering Congress and Exposition, Anaheim, CA, USA.

Korycki, R., 2006, "Sensitivity Analysis and Shape Optimization for Transient Heat Conduction with Radiation," International Journal of Heat and Mass Transfer, 49(13), 2033-2043. http://dx.doi.org/10.1016/j.ijheatmasstransfer.2006.01.007

Rauch, C., and Almbauer, R., 2010, „Uncertainty and Local Sensitivity Analysis of View Factors for Steady-State Conjugate Heat Transfer Problems, “ AIAA-2010-4907, 10 ${ }^{\text {th }}$ AIAA/ASME Joint Thermophysics Heat Transfer Conference, Chicago, IL, USA. 
Rauch, C., and Almbauer, R., 2010a, "On Uncertainty and Local Sensitivity Analysis for Steady-State Conjugate Heat Transfer Problems," IHTC14-22859, $14^{\text {th }}$ International Heat Transfer Conference, Washington DC, USA.

Siegel, R., and Howell, J. R., 2002, Thermal Radiation Heat Transfer, $4^{\text {th }}$ ed., Taylor \& Francis, New York.

Taylor, R. P., Luck, R., Hodge, B. K., and Steele, W. G., 1993, "Uncertainty Analysis of Diffuse-Gray Radiation Enclosure Problems - A Hypersensitive Case Study," NASA CP 10122, $5^{\text {th }}$ Annual Thermal and Fluids Analysis Workshop, NASA, Washington, DC, $27-40$.

Taylor, R. P., Luck, R., Hodge, B. K., and Steele, W. G., 1994, "Uncertainty Analysis of Diffuse-Gray Radiation Enclosure
Problems," AIAA 94-0132, 32 $2^{\text {nd }}$ Aerospace Sciences Meeting and Exhibit, Reno, NV.

Taylor, R. P., Luck, R., Hodge, B. K., and Steele, W. G., 1995, "Uncertainty Analysis of Diffuse-Gray Radiation Enclosure Problems," Journal of Thermophysics and Heat Transfer, 9(1), 6369.

http://dx.doi.org/10.2514/3.629

Taylor, R. P., and Luck, R., 1995, "Comparison of Reciprocity and Closure Enforcement Methods for Radiation View Factors," Journal of Thermophysics and Heat Transfer, 9(4), 660-666. http://dx.doi.org/10.2514/3.721 